Bio - grafia. Escritos sobre la Biología y su Enseñanza. ISSN 2027

Edición Extraordinaria. p.p. 856- 870

Memorias del VIII Encuentro Nacional de Experiencias en Enseñanza de la Biología y la Educación Ambiental. III Congreso Nacional de Investigación en Enseñanza de la Biología.

\title{
APROXIMACIÓN A LA INCORPORACIÓN DE LA DIMENSIÓN AMBIENTAL EN LA EDUCACIÓN SUPERIOR, CASO: UNIVERSIDAD PEDAGÓGICA Y TECNOLÓGICA DE COLOMBIA (UPTC)
}

\author{
APPROACH TO THE INTRODUCTION OF THE ENVIRONMENTAL DIMENSION IN \\ HIGHER EDUCATION CASE: PEDAGOGICAL AND TECHNOLOGICAL UNIVERSITY \\ OF COLOMBIA (UPTC) \\ Yamile Pedraza Jiménez ${ }^{1}$ \\ yamilepedraza@gmail.com
}

\section{Introducción}

Dentro del sistema educativo las instituciones de Educación superior (IES), están llamadas a debatir el qué y para qué enseñar según las necesidades y prioridades del contexto de las que hacen parte; dentro de dichas prioridades está el aportar a aquellas crisis sociales, ambientales que han surgido de la relación del hombre con lo natural. Incluir la dimensión ambiental requiere saber cómo se gestiona, cuáles son las acciones y quiénes se comprometen en procesos de la enseñanza e investigación del saber ambiental que los profesionales requieren para lograr el abordaje y proponer vías de avance a aquellas problemáticas ambientales desde la aplicación de los conocimientos con criterios éticos, posición política y en el diálogo con otros saberes, modos y formas de cultura que afectan o se ven afectados por causas ambientales.

Se describe inicialmente, los múltiples llamados y recomendaciones de diferentes cumbres, expertos y sociedad que le hacen a las IES sobre la urgencia de la incorporación de lo ambiental en ellas; luego se expone un contexto especifico de universidad pública, en la que un antecedente y otras características de organización estructural de la institución pueden ser un escenario potencial para desarrollar allí una propuesta de diseño curricular que aporte a una cultura ambiental de los futuros profesionales en todas las áreas del conocimiento, que les será útil para la toma de decisiones en su desempeño laboral.

Palabras Clave: Dimensión ambiental, currículo, Educación Superior.

Contexto conceptual: La necesidad de la Formación Ambiental

\footnotetext{
${ }^{1}$ Estudiante del Doctorado Interinstitucional en Educación de la Universidad Distrital “Francisco José de Caldas". Director: Dr. William M. Mora Penagos.
} 
Bio - grafia. Escritos sobre la Biología y su Enseñanza. ISSN 2027

Edición Extraordinaria. p.p. 856- 870

Memorias del VIII Encuentro Nacional de Experiencias en Enseñanza de la Biología y la Educación Ambiental. III Congreso Nacional de Investigación en Enseñanza de la Biología.

La educación debe convocar a la participación activa de los ciudadanos a través de un currículo, pertinente con la sociedad, brindando la posibilidad de aprender lo necesario para poder participar en ella sin tener el riesgo de ser excluido.

Pero en primera instancia es válido hacer un reconocimiento a las demandas sociales hacia el sistema educativo, entre ellas algunas planteadas desde posiciones como las de la Educación popular o de las pedagogías progresistas que buscan la transformación de las prácticas educativas y de los sujetos como parte activa de la toma de decisiones, se expone asi que se requiere un sistema educativo que realice una lectura crítica del orden social y el rol de la educación en dicho orden; se parte de la convicción que desde la educación se puede contribuir actuando sobre la subjetividad en la formación de ciudadanos, así como potenciar, generar y emplear metodologías educativas dialógicas, participativas y activas. Además se requiere recobrar la visión de la Educación como hecho autónomo crítico a las estructuras tecno científicas impuestas para mantener ideologías hegemónicas que han llevado a una crisis educativa y planetaria.

En un mundo globalizado es pertinente el surgimiento de propuestas alternativas o cambios en la educación que habiliten a los ciudadanos en los conocimientos y saberes pertinentes, que aporten a abordar y avanzar en situaciones de problemas reales y que contribuyan a mantener una actitud de aprendizaje constante para que los sujetos se construyan en beneficio de una sociedad justa y equitativa.

La educación ambiental (EA) surge como una propuesta alternativa, hace cuatro décadas, ante la complejidad e integralidad para estudiar los fenómenos y crisis ambiental que el sistema educativo hasta el momento ha fragmentado, descontextualizado o no ha tenido en cuenta en la construcción de los currículos de las instituciones educativas IE. El propósito de la EA es articular la relación de lo natural con lo social, que desde los países del sur se enmarca como una posición por lo sustentable "que promueva una nueva cultura política fundada en una ética de la sustentabilidad -en valores, creencias, sentimientos y saberesque renuevan los sentidos existenciales, los mundos de vida y las formas de habitar el planeta Tierra" (Mora Penagos, 2007).

Desde el planteamiento de la EA (Conferencia de Tibilis, 1977) ha pasado por varias perspectivas de las personas, académicos y activistas que la han asumido, aun así persisten enfoques conservacionistas y ecologistas o también aquellas buenas intenciones de la EA sin soportes pertinentes para abordar las problemáticas; falta concreción del impacto de la EA 
Bio - grafia. Escritos sobre la Biología y su Enseñanza. ISSN 2027

Edición Extraordinaria. p.p. 856- 870

Memorias del VIII Encuentro Nacional de Experiencias en Enseñanza de la Biología y la Educación Ambiental. III Congreso Nacional de Investigación en Enseñanza de la Biología.

para la formulación de política públicas y que las instituciones reconozcan su prioridad; se ha intentado introducir y remplazar la EA por el desarrollo sostenible.

Se observa además que algunas propuestas EA, desde el ecologismo ambiental, han abordado problemas que se reducen a la mercantilización de los recursos y el impacto de su escases; por lo tanto, se estudian desde áreas del conocimiento de la ciencia y la tecnología; faltando complementarse con una perspectiva histórica y cultural que propone la EA.

Existen en su mayoría referencias de trabajos y estrategias de incorporación de EA en los niveles educativos de básica, media (bachillerato, ESO) a nivel mundial, que han llevado a la conformación de políticas educativas a través de los estamentos encargados del ambiente y la educación en las diferentes naciones; pero a pesar de los esfuerzos realizados por diferentes cumbres, declaraciones y redes en educación superior la incorporación de la EA tanto a los currículos como las políticas institucionales, ha sido obstaculizada por diferentes causas; entre ellas: la estructura curricular tradicional, el desconocimiento o la indiferencia a los acuerdos internacionales, la falta de organización, compromiso y vinculación de los docentes que trabajan en el campo ambiental.

Por ejemplo, las estrategias en EA fueron objeto del Seminario Internacional de Capacitación para la Incorporación de la EA en el Currículo de la Educación Superior (Talloires, 1990), Básica Primaria (Malta, 1991) y Básica Secundaria (El Cairo, 19991). En Educación superior se siguió la discusión con la declaración de Halifax (Canada 1991) de estos encuentros surgieron recomendaciones como la participación de los docentes en el diseño de un currículo, para incorporar la dimensión ambiental en todos los planes y procesos escolares y la investigación de métodos de evaluación para estos procesos (Mora Penagos, 2007).

En igual sentido en las (Conferencia de Tibilis, 1977) (Memorias Universidad y Medio Ambiente en América latina y el caribe, 1985) "Carta de Bogotá", se proponen estrategias para incorporar la dimensión ambiental en la ES como:

- La creación de nuevas carreras ambientales a nivel de pregrado y de posgrado,

- La introducción de la dimensión ambiental en las carreras tradicionales

- la investigación ambiental,

- La interdisciplinariedad,

- Acciones de extensión ambiental universitaria, entre otras, Trelles, 2006 en (Mora Penagos, 2007) 
Bio - grafia. Escritos sobre la Biología y su Enseñanza. ISSN 2027

Edición Extraordinaria. p.p. 856- 870

Memorias del VIII Encuentro Nacional de Experiencias en Enseñanza de la Biología y la Educación Ambiental. III Congreso Nacional de Investigación en Enseñanza de la Biología.

- En el campo del conocimiento se planteó el concepto de dimensión ambiental como un recurso de análisis teórico y político para que las universidades logren apoyar las propuestas globales, González, 2000 en (Mora Penagos, 2007).

Además "la formación de redes universitarias hacia la sostenibilidad", en las que se destacan: Aces, Complexus, Oiudsma, Suma 21, Cubana de Formación Ambiental; La Red Aces (formada por 11 universidades, 5 europeas y 6 Latinoamericanas) en Iberoamérica; la Red Latinoamericana de Formación Ambiental de la que hace parte Colombia (Mora Penagos, 2007).

En diciembre de 2013, se adelantó el primer Foro Latinoamericano de Universidades y Sostenibilidad, en Viña del Mar (Chile) coordinado por la Alianza de Redes Iberoamericanas de Universidades por la sustentabilidad y el Ambiente (ARIUSA), la Red de Formación Ambiental para América latina y del Caribe (RFA-ALC) y el Capitulo Latinoamericano de la Alianza Mundial de universidades sobre Ambiente y Sostenibilidad (GUPES-LA) el cual tuvo como objetivo discutir y evaluar el avance en la construcción indicadores de sustentabilidad de las universidades en América Latina. Este Foro, congregó a un número significativo de universidades (45), Redes universitarias ambientales (19) y otras representaciones universitarias, participaron un total de 14 países entre ellos Colombia; se propone alli que dada la diversidad y no buscando establecer un solo modelo, adelantar el Proyecto de la Rede de Indicadores de Sustenibilidad Universitaria RISU (Benayas, 2014. p 14).

Dicho proyecto RISU ha propuesto 11 dimensiones sobre las cuales se definen indicadores de evaluación de la sustentabilidad en las universidades latinoamericanas, así: Política de sostenibilidad (15 indicadores); Sensibilización y participación (12 indicadores); Responsabilidad socioambiental (10 indicadores); Docencia (13 indicadores); Investigación y transferencia (13 indicadores); Urbanismo y biodiversidad ( 7 indicadores); Energía (10 indicadores); Agua (10 indicadores); Movilidad (8 indicadores); Residuos (11 indicadores) y Contratación responsable (5 indicadores) $)^{2}$.

Según el informe de la Red de Indicadores de sustentabilidad en universidades (RISU, 2014) a partir de encuestas aplicadas a 65 universidades de 10 países latinoamericanos, la

\footnotetext{
${ }^{2}$ http://ariusa.net/es/publicaciones/informe-sobre-resultados-del-proyecto-risu
} 
Bio - grafia. Escritos sobre la Biología y su Enseñanza. ISSN 2027

Edición Extraordinaria. p.p. 856- 870

Memorias del VIII Encuentro Nacional de Experiencias en Enseñanza de la Biología y la Educación Ambiental. III Congreso Nacional de Investigación en Enseñanza de la Biología.

dimensión de docencia pretende evaluar el grado de integración de la formación en sostenibilidad en la oferta académica de las universidades, analiza si los currículos de diferentes carreras que incorporan contenidos de sostenibilidad para fomentar un cambio en las actitudes en los futuros profesionales al aplicar un enfoque holístico a la hora de abordar y platear soluciones a problemas ambientales.

Así el informe RISU (2014) dentro del núcleo de docencia muestra puntos débiles, como: solo un 5\% de las universidades tiene alguna herramienta para evaluar el aprendizaje en sustentabilidad; un $9 \%$ de centros existe alguna comisión o grupo técnico encargado de asesorar a los centros en la adaptación de los currículos para introducir en ellos criterios de sustentabilidad; el 20\% existen estrategias de formación y actualización de docentes para fortalecer la perspectiva ambiental y de sustentabilidad en la docencia y en los planes de estudio. Puntos fuertes: Por encima de un $50 \%$ existe mención expresa a las actividades docentes en la política de sustentabilidad de la universidad; el $45 \%$ de las universidades al menos en el $10 \%$ de los currículos académicos de las distintas carreras se incorpora la perspectiva de la sustentabilidad y un $37 \%$ de la muestra se realiza proyectos de fin de carrera o trabajos de posgrado relacionados con la sustentabilidad en la universidad

Si bien las cumbres han impactado las prácticas ambientales, en las instituciones universitarias falta más apoyo por parte de estas entidades, se plantea que una de las soluciones, podría ser incluir los indicadores de sustenibilidad y rendimiento de cuentas a la sociedad en los procesos de autoevaluación con implicaciones políticas y económicas para su actividad (Mora Penagos, 2007); o el evaluar hacia dónde va cada propuesta curricular escrita de las IES (en un contexto especifico) y contrastar con su realidad, lo que indicará cómo se construyen estas instituciones como universidades sustentables.

(Sauvé, 2014), en lugar de referirse a indicadores (por su aplicación económica), los determina criterios: uno de ellos parte de caracterizar el objeto de estudio de la EA, porque no es el ambiente, ya que el saber ambiental no se limita a la construcción de conocimiento, "el propósito de la EA es la relación de lo social con el ambiente"; el criterio de valoración sería ver más allá de los saberes qué se hace con ellos en el actuar ciudadano. Educar quiere decir acompañar a cada uno en su proyecto de vida, hay conocimientos que son muy importantes en el campo científico que son de debate profesional y políticamente valorizados, pero raramente, por ejemplo, se le oye a un político un discurso ético o ecosófico en defensa de una mejor convivencia con nuestro planeta. 
Bio - grafia. Escritos sobre la Biología y su Enseñanza. ISSN 2027

Edición Extraordinaria. p.p. 856- 870

Memorias del VIII Encuentro Nacional de Experiencias en Enseñanza de la Biología y la Educación Ambiental. III Congreso Nacional de Investigación en Enseñanza de la Biología.

Como se puede observar, en la actualidad el sistema educativo se le han atribuido nuevas demandas sociales, incluyendo aquellas que tienen que ver con las decisiones frente a la naturaleza que lo rodea y sus implicaciones sociales y ambientales; en este panorama se requiere repensar cuáles son los fines prioritarios de la educación y cómo colaborar o compartir responsabilidades con otras entidades sociales y comunitarias es decir equé se aprende? Y ¿para qué? en respuesta un mundo globalizado.

Dentro del campo problémico de la incorporación de la EA en los diferentes niveles educativos encontramos la necesidad de la transdisciplinaridad que requiere el abordaje de las causas que han conducido a la crisis planetaria; la EA es considerada como un metadiscurso de origen tanto en las ciencias naturales como en las ciencias sociales; a pesar de ello, la dificultad ha estado en encontrar la articulación y aplicabilidad de dicho discurso en avanzar hacia soluciones reales causados por la relación inequitativa de la acción social sobre el mundo natural, se ha delegado esta responsabilidad en el mayor de los casos en las IE, a la disciplina de la ecología o en general al campo de las ciencias naturales limitando el ambiente a una visión de relaciones factores bióticos y abióticos o asumiendo la crítica a las implicaciones ambientales de la ciencia y la tecnología y si bien estos aspectos los debe asumir una $E A$, influyen otras variables de orden social en la toma de decisiones, como la formación política, económico, cultural, crítica y ética de los ciudadanos; se refleja entonces la necesidad de construir currículos pertinentes y alternativos para las regiones de influencia de las entidades educativas.

En Colombia el profesor Ángel Augusto Maya expuso las relaciones entre campos como la ecología, historia, filosofía, ética, estética geografía y educación e hizo un llamado a la reflexión a comprender lo ambiental desde perspectiva política, social y cultural, expuso que la ecología como ciencia de los ecosistemas no es suficiente para comprender la actividad humana ya que para este autor el hombre no posee un nicho ecológico y su hilo de contacto con lo natural son las distintas formas como la sociedad humana interactúa con los ecosistemas a lo que denominó "cultura" o formas de adaptación del hombre a la naturaleza, en consecuencia el actuar hacia ella depende de su cultura, el "hombre se mueve entre leyes de la naturaleza y las de la cultura" (Noguera, 2009)

Dentro de los procesos dinámicos de las IES que incluyen la investigación, docencia y extensión para la generación de conocimiento, la apropiación cultural a través del uso la democracia y la participación, la academia solicita herramientas desde la 
Bio - grafia. Escritos sobre la Biología y su Enseñanza. ISSN 2027

Edición Extraordinaria. p.p. 856- 870

Memorias del VIII Encuentro Nacional de Experiencias en Enseñanza de la Biología y la Educación Ambiental. III Congreso Nacional de Investigación en Enseñanza de la Biología.

interdisciplinariedad y la interculturalidad entre otras, para la reflexión crítica sobre las formas de concebir el desarrollo de un país que se reflejen propuestas curriculares alternativas ambientalmente sustentables, así como Angel Maya lo expresaba:

"Superando las visiones restringidas que interpretan lo ambiental como un problema ecológico o exclusivamente tecnológico, esta propuesta intenta comprenderlo como un objeto de estudio en todas las disciplinas científicas, desde las ciencias naturales y tecnológicas, hasta las ciencias que estudian el comportamiento humano. El problema ambiental es responsabilidad de todos". (El reto de la vida. 1996 en (Noguera, 2009). Lo ambiental debe ser objeto y sujeto de estudio de todas las disciplinas.

Las IES están en el deber de ser y ofrecer desde su propuesta curricular a la comunidad acompañamiento y soluciones pertinentes, mediados por profesionales que se han construido en escenarios académicos con profesores, investigadores y pares en constante critica que les permiten enfrentar con capacidad, competencia y responsabilidad las realidades ambientales.

Las universidades tienen como función de favorecer modelos de sustentabilidad ambiental, además de la producción de conocimiento estas entidades tienen el objetivo político de solucionar problemas de la sociedad, a través de la responsabilidad de formación de profesionales que contribuyan a abordar problemas complejos e interdisciplinares propios de relaciones ciencia / tecnología - sociedad / ambiente (Mora Penagos, 2007).

Los escenarios con los que se cuenta en las IES para abordar la dimensión ambiental van desde áreas de conocimiento en diferentes programas académicos, proyectos de bienestar universitario y el trabajo con la comunidad; estos escenarios requieren ser articulados para aportar en la formación de sujetos comprometidos y lograr la incorporación "en el currículo la dimensión ambiental, para desarrollar conceptos tales como el riesgo, la responsabilidad social, la equidad, la salud ambiental, el desarrollo, la conservación, la calidad de vida, la investigación - acción, la interculturalidad, la gobernabilidad y la ética universal como base de un dialogo permanente entre el Estado y la Universidad, entendida ésta como el cerebro-centro de la sociedad en evolución" (Política Nacional Ambiental); esto implica que para llevar a cabo cualquier propuesta educativa en las IES, ya sea a nivel de pregrado o posgrado, se puede trabajar en la concertación y cogestión con los diferentes integrantes de la comunidad académica reconociendo los límites y alcances de este trabajo conjunto y las intecionalidades del proyecto en común. 
Bio - grafia. Escritos sobre la Biología y su Enseñanza. ISSN 2027

Edición Extraordinaria. p.p. 856- 870

Memorias del VIII Encuentro Nacional de Experiencias en Enseñanza de la Biología y la Educación Ambiental. III Congreso Nacional de Investigación en Enseñanza de la Biología.

En consecuencia, el inicio de una práctica de la cultura ambiental requiere del compromiso acertado de docentes que a su cargo tienen en las IES el campo ambiental y esto incluye a programas de formación de profesionales de las áreas disciplinares de las ciencias naturales y de las ciencias sociales que contribuyen a la formación del ser fundamental de las personas, bajo la filosofía de que el ambiente nos forma y transforma de la misma manera que lo transformamos (comunicación personal (Sauvé, 2014) refiriéndose a la eco formación). Por tanto si el rol del profesor es potencializar el desarrollo de competencias y habilidades en la formación integral de los ciudadanos es imprescindible valorar sus propias capacidades y competencias.

La cultura ambiental facilitaría la articulación entre estados disyuntos de la universidad: misión académica / compromiso social, competitividad / cooperación, especialización / interdisciplinariedad, enseñanza / investigación, dualidades que pasarían a ser elementos unificadores de la actividad universitaria a través de una formación epistemológica que permita unir lo que la modernidad separó (Mora Penagos, 2007).

Existe así dentro de los desafíos de las IES realizar una formación continua de sus docentes en los diferentes programas universitarios, fomentar prácticas y proyectos ambientales en que los estudiantes participen, compromiso y seguimiento a proyectos ambientales institucionales $e$ interinstitucionales con participación de la comunidad y promover investigación en EA según las necesidades específicas de contexto local o nacional.

Para (Leff, 1997) las instituciones de educación superior tienen como reto frente a la educación ambiental no sólo ampliar la concientización en las diferentes disciplinas del conocimiento sobre los problemas ambientales, sino el de dar una mayor eficacia al proceso de la educación ambiental, generar nuevos conocimientos teóricos y prácticos y asegurar que éstos se incorporen a programas de investigación y de formación evaluados y legitimados por éstas.

Educar para la vida, como condición fundamental, educación sobre el ser, saber, saber hacer $y$ el saber vivir juntos, educar para el presente no para el futuro desarrollar capacidades para la vida y aprendizajes más activos y permanentes que vinculen la Trandisciplinaridad y la cultura, la aplicación de lo disciplinar con el entorno, debatir sobre lo ambiental es un derecho de todo integrante de la comunidad educativa por lo tanto la EA es un deber de las IES proporcionando los espacios para su incorporación (Mora Penagos, 2012). 
Bio - grafia. Escritos sobre la Biología y su Enseñanza. ISSN 2027

Edición Extraordinaria. p.p. 856- 870

Memorias del VIII Encuentro Nacional de Experiencias en Enseñanza de la Biología y la Educación Ambiental. III Congreso Nacional de Investigación en Enseñanza de la Biología.

\section{Contexto}

Algunas Universidades del país han asumido a paso lento las recomendaciones resultado de las cumbres y de las políticas nacionales la incorporación de la EA; la Universidad Pedagógica y Tecnológica de Colombia (UPTC) es una de ellas, en sus fundamentos misionales y visionales se ha comprometido con la formación de ciudadanos integrales y transformación social con pilares en los valores éticos, culturales y las bondades de la ciencia y la técnica. Además, se resalta una posición en función de un proyecto educativo a favor del desarrollo sostenible

En su función social se compromete con el ofrecimiento de programas formales profesionales y disciplinares, en los niveles de pregrado, postgrado y de formación permanente, que hacen efectivos los derechos humanos individuales, colectivos y culturales pertinentes para el desarrollo económico y ecológico de la nación, y la permanente observación de los adelantos tecnológicos y su asimilación prioritaria para la consolidación de una sociedad con bienestar y desarrollo social.(Plan Maestro)

La UPTC cuenta con 11 facultades: Ciencias agropecuarias, ciencias básicas, Ciencias de la educación, ciencias económicas y administrativas, ciencias de la salud, derechos y ciencias sociales, ingeniera, sede Chiquinquirá, sede Duitama, sede Sogamoso y la Facultad de estudios a distancia.

Dentro de los programas de posgrado que tiene nociones del componente ambiental en su currículo encontramos un programa de especialización en Gestión ambiental en la sede Sogamoso, Maestría y especialización en Ingeniería ambiental, especialización en calidad y seguridad alimentaria, Maestría en ciencias agrarias, ciencias biológicas, Desarrollo rural, derechos humanos, Educación y patrimonio cultural.

En el Pregrado cuenta con el Programa de Licenciatura en Ciencias Naturales y Educación Ambiental, que por su nominación en su propuesta curricular, la misión, visión, justificación, propósitos, perfil profesional y ocupacional, investigación, extensión y plan de asignaturas ha incluido la formación en EA, otras licenciaturas Prescolar y sociales que incluyen en sus programas nociones ambientales a través de asignaturas (Medina Peña, 2011). Ahora programas académicos de pregrado como la Ingeniería agronómica, Biología, Química, 
Bio - grafia. Escritos sobre la Biología y su Enseñanza. ISSN 2027

Edición Extraordinaria. p.p. 856- 870

Memorias del VIII Encuentro Nacional de Experiencias en Enseñanza de la Biología y la Educación Ambiental. III Congreso Nacional de Investigación en Enseñanza de la Biología.

economía, medicina, derecho, administración en empresas agropecuarias, Ingeniería y diseño industrial, incluyen asignatura una o dos asignaturas que relacionan con el ambiente como desarrollo sostenible, ambientes saludables, ecología, derecho ambiental, ecología humana, química ambiental, agroecología entre otras, por su parte las Ingenieras: geológica, minas, civil, transportes y vías en sus estructuras académicas incluyen todas aquellas que sean de planeación, extracción, optimización de recursos en función del desarrollo económico del país y propuesta de formación de los sujetos en desde la proyección del desarrollo sostenible (ver Misiones y visiones página Web Uptc).

El programa de Ingeniería ambiental, dentro de sus propósitos misionales prepara profesionales integrales, de visión interdisciplinaria, con pensamiento crítico capaces de diagnosticar y proponer soluciones a problemas ambientales en su contexto y la nación con el uso de los conocimientos científicos y técnicos con criterios de equidad, calidad de vida, enfoque sostenible con respeto a la cultura y el medio ambiente (http://www.uptc.edu.co/). La UPTC no ha mostrado voluntad de hacer parte de la red de universidades colombinas sostenibles, convocadas por ASCUN.

\section{Antecedente Institucional}

Se ha adelantado un estudio de Maestría, sobre cómo la EA se ha incorporado en los programas de pregrado de la Facultad de Ciencias de la Educación (FCE) de La UPTC, a través de análisis del documento de programa académico educativo PAE de cada programa (misión, visión, propósitos, objetivos, características de perfil profesional, ocupacional, competencias, organización y distribución de las diferentes áreas); la aplicación de encuestas a estudiantes de último semestre y entrevista a las directivas de la FCE y establece elementos necesarios para la elaboración de una propuesta de inclusión de la EA en la Facultad en función que los futuros profesionales demandan una formación en escenarios que contribuyan al diálogo de saberes entre comunidades, posibilitando una formación alternativa con valores éticos y estéticos que permitan la convivencia y su relación con lo natural (Medina Peña, 2011), La propuesta planteada se escribe en función de la Política Nacional Ambiental para Colombia que es conocida por su tendencia al desarrollo sostenible.

Según la investigación titulada "La EA en los programas académicos de la FCE de la UPTC" (Medina Peña, 2011) se reportan resultados como: 
Bio - grafia. Escritos sobre la Biología y su Enseñanza. ISSN 2027

Edición Extraordinaria. p.p. 856- 870

Memorias del VIII Encuentro Nacional de Experiencias en Enseñanza de la Biología y la Educación Ambiental. III Congreso Nacional de Investigación en Enseñanza de la Biología.

La incorporación EA en la universidad se ha reducido a la inclusión de asignaturas de carácter ambiental o ecológico, de los 12 programas de la facultad en su PAE reporta que sólo un $25 \%$ presentan nociones de formación de ambiente, se evidencia el hecho de no acatar las recomendaciones desde las cumbres ambientales y de política nacional ambiental en la inclusión de la EA. Se resalta que la formación de licenciados en Ciencias Naturales y Educación Ambiental, Prescolar y Sociales incluyen la noción ambiental en sus misiones, visiones y designan en su programas asignaturas para abórdala (Medina Peña, 2011).

En lo referente a la asignatura Cátedra Universidad y Entorno, que se incluyó en el área general, a partir de la reforma académica realizada por la UPTC, es una asignatura que cuenta con tres créditos académicos, uno de ellos dedicado al Entorno. Revisado el propósito de esta asignatura, se encontró que se abordan aspectos superficiales en lo concerniente a la EA, pues sólo se le dedica una tercera parte del tiempo total de la asignatura durante el semestre, y como tal se hace a través de temas que de forma particular se abordan en tiempos específicos, esto sin contar con docentes preparados para desempeñar esta función (Medina Peña, 2011).

En cuanto al análisis estratificado de la encuesta a 60 estudiantes, 5 de cada programa que cursan el último semestre a 2009 , un $72 \%$ manifestó no tener formación en EA durante su carrera; pero el porcentaje aumenta en cuanto a las exigencias sociales de su práctica: el $47 \%$ manifiesta haber desarrollado actividades con nociones ambientales; el cuestionamiento aquí sería, ¿cómo las asumieron, desde qué posiciones, contaban con los elementos necesarios, se convirtió en un activismo, consideran que hubo aporte o no de su universidad? Sobre si consideran que las nociones ambientales deben ser asumidos por los licenciados en Ciencias Naturales y Educación Ambiental, un $98 \%$ no considera que debe ser así, argumentan que todos deben formar una conciencia ambiental independiente de la profesión que se tenga, así como que la formación ambiental involucra al humano por tanto es de formación esencial y es necesario tener formación en lo EA para ponerlo en práctica (Medina Peña, 2011).

El autor del trabajo realizado en la FCE de la UPTC sobre la incorporación de la EA, discute que se hace necesario generar nuevos conocimientos, saberes que se incorporen a procesos de investigación y de formación y que se evidencien al finalizar el paso de los estudiantes por la universidad, el esfuerzo debe ir más allá, propiciar escenarios de diálogo, reconocimiento e interpretación [acción] de dinámicas para articular lo social natural en la formación "integral" de los futuros profesionales. Esta dinámica requiere de 
Memorias del VIII Encuentro Nacional de Experiencias en Enseñanza de la Biología y la Educación Ambiental. III Congreso Nacional de Investigación en Enseñanza de la Biología.

proyectar estrategias que permitan la posibilidad de construirse en una cultura de lo ambiental, busque la aplicación del conocimiento en investigación entorno a lo ambiental; que articule áreas, sea efectiva, participante y transformadora que incluya la formación de talento humano en la temática ambiental a nivel pregrado y posgrado (Medina, 2011). Todo saber está para ser cambiado, modificado y entrelazado con otros saberes: la percepción del mundo como un mundo de complejidad, Leff, 1997 en (Medina Peña, 2011).

La investigación realizada en la FCE de la UPTC, realiza una propuesta para la incorporación de la EA en los 12 programas de dicha Facultad; tiene en cuenta procesos o fases que incluyen el trabajo con directivos, la creación de un grupo de trabajo para la incorporación de la EA (GTIEAFCE) constituido por directores y profesores interesados de cada programa en esta intención quienes se encargarían de estructurar, tomar decisiones a través de dinámicas como el registro de relatorías, espacios concertados de trabajo incluidos y autorizados por directivos y finaliza con una propuesta que incluye el trabajo con estudiantes (Medina Peña, 2011).

\section{Propuesta}

Como se puede inferir la UPTC, cuenta en su estructura académica con un número apreciable de programas de pregrado y posgrado y disciplinas desde las áreas científico, técnicas, socio-humanísticas y pedagógicas que pueden aportar en la articulación de programas para la formación de una cultura ambiental del buen vivir y de ciudadanos responsables, si bien se requieren cambios, por ejemplo el viraje de una visión del desarrollo sostenible hacia una visión sustentable, es preciso iniciar y continuar con el dialogo y dinámicas de acción que puedan evidenciar en forma concreta cómo se realizan procesos de dinamización de la incorporación de la dimensión ambiental en estas instituciones, donde se articula lo natural con lo social, en un contexto inicialmente interdisciplinario buscando la transdisciplinariedad al caracterizar casusas de la situación ambiental de la región como campo problémico que hagan converger el diálogo de diferentes conocimientos, saberes metodologías, posiciones, culturas etc.

Se propone a partir de lo expuesto y avanzando con el diagnóstico preliminar realizado en la FCE de la UPTC, el diseño de una oferta curricular para pregrado que surja de las discusiones de un grupo interdisciplinario de docentes e investigadores provenientes de las

diferentes facultades de la Institución quienes tomarán decisiones de cómo incorporar lo ambiental en ES y que tengan como preocupación la relación cultural de su quehacer con el mundo natural. 
Bio - grafia. Escritos sobre la Biología y su Enseñanza. ISSN 2027

Edición Extraordinaria. p.p. 856- 870

Memorias del VIII Encuentro Nacional de Experiencias en Enseñanza de la Biología y la Educación Ambiental. III Congreso Nacional de Investigación en Enseñanza de la Biología.

Dado que la UPTC no cuenta con una propuesta transversal sobre lo ambiental en el pregrado, esta opción puede contribuir a aportar elementos mínimos en éste campo que todo profesional debe incluir en su cultura; como valor agregado, se puede decir, que luego de haber cursado diez semestres en alguna área del conocimiento, buscará cómo integrar ésta en la articulación de lo social -natural. Esto adquiere importancia dado que en cuanto a la oferta laboral existen profesionales de todas las áreas que laboran en el campo de lo ambiental, que requieren una aproximación desde sus propias disciplinas a las relaciones de estos conocimientos con el mundo que habitan.

A diferencia de la propuesta realizada para la FCE el grupo de trabajo será interdisciplinario e involucrará al menos tres facultades de la UPTC y estará conformado con la selección de docentes que tengan formación de Maestría en el campo ambiental o procesos de investigación con aportes a la EA, por poseer saberes y conocimientos, procedimientos y actitudes, resultado de la reflexión en su formación investigativa en lo ambiental para orientar de manera acertada a otros.

Al igual que la propuesta realizada por (Medina Peña, 2011), a la FCE de la UPTC, tendrá fases de trabajo administrativo, de diseño e implementación de la propuesta; pasando por las discusiones y aprobaciones de orden institucional. Los directivos darán la viabilidad y establecerán criterios de las formas de trabajo y tiempos de profesores de planta de la UPTC que participen en el diseño de la propuesta. El documento producto de dicho diseño para pregrado será presentado ante el Consejo Académico, quien analizará su viabilidad dentro del área general que se oferta a todos los programas o las formas de incorporación de la dimensión ambiental en las diferentes facultades y programas, según recomendaciones del grupo interdisciplinario.

\section{Aproximación investigativa}

Se adelantará paralelamente a la declaración de la propuesta y de su proceso de desarrollo, una investigación Doctoral (Doctorado Interinstitucional de Educación), en la línea de investigación de incorporación de dimensión ambiental en Educación superior, específicamente en la formación docente.

La pregunta orientadora será ¿Qué sentidos dan los sujetos (grupo de profesores e investigadores de la UPTC) a lo ambiental en el proceso de su incorporación en el diseño curricular de la UPTC? 
Bio - grafia. Escritos sobre la Biología y su Enseñanza. ISSN 2027

Edición Extraordinaria. p.p. 856- 870

Memorias del VIII Encuentro Nacional de Experiencias en Enseñanza de la Biología y la Educación Ambiental. III Congreso Nacional de Investigación en Enseñanza de la Biología.

El proceso investigativo será orientado por la investigación cualitativa, bajo el paradigma interpretativo y se utilizará el estudio de caso de un grupo focal; el análisis y discusión se realizará a través de los datos arrojados en el discurso de los participantes, estableciendo categorías y haciendo su triangulación.

\section{A manera de Conclusión}

El saber ambiental se convierte en un derecho de todo ciudadano de estar informado, para conocer, actuar y tomar decisiones con criterios éticos, críticos y justos sobre sobre su relación con el ambiente.

Las IES tienen que proporcionar acceso a estos discursos y brindar a través de sus currículos ejemplos de cómo llevar la teoría que respalda el saber ambiental y evidenciar cómo se dan las dinámicas de las realidades al pretender la interdisciplinaridad y sistematicidad que reclama el abordaje de las problemáticas ambientales.

El avance en la investigación de la EA, requiere de estudios que en la acción que puedan contrastar teoría y práctica, para volver a resignificar la teoría, contrastar metodologías y experticia en la investigación sobre y con colectivos que pretenden aportar a las problemáticas ambientales.

\section{Bibliografía}

Conferencia de Tibilis. (1977). informe final.

Leff, E. (1997). La Educación Ambiental. II Congreso de Educaión Ambiental, sin publicar.

Medina Peña, N. A. (2011). La Educación Ambiental en los Programas Académicos de la Facultad de Ciencias de la Educación de la UPTC. Tunja: Tesis.

Memorias Universidad y Medio Ambiente en América latina y el caribe. (1985). Carta a Bogotá. Bogotá.

Mora Penagos, W. M. (2007). Inclusión de la dimensión ambiental en programas curriculares de educación superior: Un estudio entorno a las ideas del profesorado. Sevilla: Diploma estudios avanzados DEA Doctorado Interinstitucional de Educación Ambiental.

Mora Penagos, W. M. (2007). Respuesta de la Universidad a los problemas socioambientales: La ambientalización del currículo en Educación Superior. En E. S. 
Bio - grafia. Escritos sobre la Biología y su Enseñanza. ISSN 2027

Edición Extraordinaria. p.p. 856- 870

Memorias del VIII Encuentro Nacional de Experiencias en Enseñanza de la Biología y la Educación Ambiental. III Congreso Nacional de Investigación en Enseñanza de la Biología.

(Comp.), Las Ciencias Ambientales: Una nueva área del conocimiento. Bogotá: Universidad Distrital.

Mora Penagos, W. M. (2012). Educación en ciencias y educación ambiental: necesidad de una relación mutuamente beneficiosa. EDUCyT, 134-148.

Noguera, P. (2009). Augusto Angel Maya: Poeta - Filósofo del pensamiento ambiental latioameticano. ISEE, N 6. Sección Filosofia ambiental sudamericana, 1-9.

Sauvé, L. (2014). Relación entre la Ecociencia y la Educaión Ambiental [Grabado por Y. Pedraza]. Bogotá, D,C, Colombia. 